\title{
Labyrinthe
}

9| 2001

Numéro 9

\section{Phrixos, d'Euripide}

Fragments traduits du grec ancien et commentés par Laurent Dubreuil

\section{Laurent Dubreuil}

\section{(2) OpenEdition}

Journals

Édition électronique

URL : http://journals.openedition.org/labyrinthe/1120

DOI : $10.4000 /$ labyrinthe. 1120

ISSN : 1950-6031

Éditeur

Hermann

Édition imprimée

Date de publication : 30 juin 2001

Pagination : 93-101

Référence électronique

Laurent Dubreuil, «Phrixos, d'Euripide », Labyrinthe [En ligne], 9 | 2001, mis en ligne le 11 février 2006,

consulté le 21 avril 2019. URL : http://journals.openedition.org/labyrinthe/1120 ; DOI : 10.4000/

labyrinthe. 1120

Ce document a été généré automatiquement le 21 avril 2019

Propriété intellectuelle 


\section{Phrixos, d'Euripide}

Fragments traduits du grec ancien et commentés par Laurent Dubreuil

\section{Laurent Dubreuil}

Des quatre-vingt-douze pièces qu'Euripide (480-406) est censé avoir écrites, la tradition ne nous en a légué que dix-huit, sans doute un peu corrigées mais pas trop mutilées. Il faut ajouter à cela tous les fragments, beaucoup de fragments : morceaux de vers déchiffrables sur les bouts de papyri, citations alléguées dans tel traité, passages figurant dans les florilèges. Depuis quinze ans, la recherche s'intéresse de plus en plus aux tragédies défaillantes, on reprend les grandes éditions des Fragmenta, on fouille les bibliothèques, on fait parler les catalogues, on interroge les grammairiens antiques. Il en sort bien souvent des éditions nouvelles, consacrées à une tragédie inédite (et pour cause), qu'on reconstitue ad hoc. Ces superbes résurrections sont toutes utiles à la mémoire d'Euripide, même si elles témoignent d'un souci excessif accordé à l'intrigue, et, par là, au mythe du muthos grec.

2 Dans ces pages, je propose une autre approche. Voici une traduction des fragments significatifs de Phrixos que recense l'édition classique de Nauck ${ }^{1}$. Cette tragédie (ou ces deux tragédies, si l'on admet l'hypothèse qu'Euripide s'y soit repris à deux fois), sous son état fragmentaire, mérite l'attention. D'abord, elle contient, à côté de sentences plus banales, plusieurs morceaux d'un grand intérêt, révélateur de la pensée euripidienne. Ensuite, elle renvoie à des épisodes si peu fameux que nous éviterons sans peine les surimpositions mythologiques. Et je ne dirai presque rien, donc, de l'histoire ${ }^{2}$. Nous aurons ainsi les coudées franches pour lire non pas tout à fait une pièce inconnue d'un tragique grec, mais ce qu'est devenue pour la postérité une œuvre d'Euripide.

3 Les fragments qui suivent sont tous traduits et commentés par mes soins. C'est le sens de l'interprétation qui justifie l'ordre d'apparition des textes. Je suis le texte de l'édition de Nauck et indique sa numérotation des fragments. À ma connaissance (faillible) et avant la parution du dernier livre des Fragments d'Euripide aux Belles Lettres, aucune traduction française de Phrixos n'existe en volume.

4 Cette traduction veut rendre avant toutes choses ce qui est généralement omis par les habitudes universitaires. Le rythme, les nombreux effets phoniques, les sous-entendus verbaux ne se séparent pas de la pensée d'Euripide, qu'on aurait tort, au passage, de 
réduire à un verbiage obscur. Cela m'a contraint à quelques modifications, sans souci de la bienséance des "études anciennes ». J'ai reçu l'aide précieuse de Laurent Ferri et de Sylvère Guiriec. Malgré mon travail et le soutien de l'amitié, je suis certain que des améliorations ou des corrections sont encore possibles; j'en laisse l'initiative aux lecteurs, qui doivent prendre cette publication comme un premier essai.

1. Traité sur la géopolitique

Une fois que Cadmos délaissa la ville de Sidon, l'enfant d'Agénor vint en terre thébaine. Né phénicien, il opta pour le peuple grec à la place, en habitant le pays de Dircè. Par quelle nécessité il vint et laissa les plaines de Phénicie, je pourrais le dire. Il y avait les trois garçons d'Agénor : Cilix, d'après qui l'on appelle ainsi la Cilicie, Phénix - c'est donc son nom que porte la région -, et Thasos [...]. (Fr. 8193)

5 Par originalité, nous commençons par le commencement. Les tragédies grecques ne manquent pas de reprendre l'histoire familiale pour bien préciser ce qui va se passer, quitte à remonter assez haut, et, en l'occurrence, bien avant la naissance de Phrixos. À mon sens, il ne s'agit pas vraiment de redites ritualistes ou d'une occasion de rafraîchir la mémoire des auditeurs. Dans ces expositions, le poète commence à construire le mythe dont il a besoin.

6 Euripide évite ici le simple aspect litanique des généalogies. Il présente une préhistoire politique. Les fils de l'archaïque Agénor sont les grandes figures de la territorialisation, les voyageurs finissant par se fixer. Phénix, Cilix et Thasos sont des noms de pays à eux seuls. Dans ce passage, Cadmos change de sol, et, c'est son droit, il change de peuple. Dans les stratégies indigènes du protonationalisme, que la traduction fait volontairement ressortir, le Grec Euripide raconte l'hellénisation de Cadmos. On peut toujours imaginer, comme on le racontait dans l'Antiquité, que Cadmos introduisit l'alphabet ; on peut rappeler que Cadmos passe pour avoir créé Thèbes. Mais dans sa description des fondations, la pièce mélange le temps lointain et la situation contemporaine. Cadmos est né phénicien, du nom de son frère, et celui qui établira Thèbes arrive dans une terre qu'on qualifie par avance de thébaine. C'est le paradoxe géopolitique : il faut admettre que le Phénicien s'est transformé en Grec en arrivant dans un royaume hellénique si l'on veut éviter qu'un Phénicien ait fondé une ville grecque 4

Car les cités ce sont des hommes, pas des déserts.

Car les cités ce sont des hommes, pas des habitations. (Fr. 828)

7 Les deux lignes sont les deux versions d'un même vers, qui prolonge nos considérations politiques dans des directions différentes. Suivant la leçon qu'on choisit pour le dernier mot (« déserts » ou « habitations »), on tirera des enseignements complémentaires. Pour la première phrase, la population des citoyens implique un fonctionnement commun. L' èrémia est aussi un désert au sens classique, un lieu à l'écart, où se retirer en solitaire. Rien à voir, donc, avec la communauté politique de la polis. Laquelle n'est pas une collection objectale et matérielle, mais tout simplement des hommes vivants - comme le confirme la seconde version.

\section{Aide-mémoire de morale}

8 De l'aphorisme politique, nous passons aux conseils moraux, prêts à l'emploi dans la vie courante.

Justement dit : avec le mari qu'elle épouse, une femme doit, dans la communauté, supporter toujours les coups du sort. (Fr. 823)

Une femme pour un mari dans les malheurs et les maux est chose des plus agréables, si elle tient bien sa maison, elle apprivoise la colère, et délivre l'âme de l'affliction [...]. (Fr. 8225) 
Les belles-mères, à ce qu'on dit, ont des intentions malsaines pour les enfants issus

d'autres mariages, et je me garderai de les en blâmer. (Fr. 8246)

Les trois exemples que je regroupe ici nous en disent au moins autant sur l'origine de leur préservation que sur la consistance de leur sens. La plupart des fragments de Phrixos viennent des anthologies de Stobée. On a ainsi gardé beaucoup de sentences en vue d'édifier les masses. Méritaient-elles plus que d'autres, perdues, de rester jusqu'à nous ? Nous payons les frais d'une transmission assurée par les bonnes âmes.

La première salve est destinée aux femmes, pour les exhorter à rester tranquilles. Ces paroles raisonnables sont fort courantes chez Euripide, et, à mon avis, le plus souvent discréditées. Elles font bien dans la bouche des vieillards indignes, des traîtres ou des gentilles nourrices résignées. Si l'on ne veut pas conclure ainsi (et trop rapidement, peutêtre), on soulignera quelques détails intéressants.

11 Le fragment 823 dessine les contours de la communauté conjugale où la femme (comme l'enfant et les esclaves) dépend d'un viril despôtès, une situation qu'entérine et justifie abondamment la philosophie par la suite et qui n'est pas vraiment la république des égaux. Le $\mathrm{n}^{\circ} 822$ délivre dans ce cadre un conseil pour la bonne tenue de sa maison.

Le fragment 824 reprend le sujet de la marâtre. Il vaut la peine de noter que l'idée, au demeurant banale, est présentée comme un dicton. Les énoncés triviaux, dont on a parfois fait grief à Euripide, sont souvent dans ses pièces des prétextes allégués par les personnages pour se couvrir. Le sens commun est parfois juste ; et parfois ne sert qu'à camoufler ses propres faiblesses. Le tragique se livre donc fréquemment à un examen des opinions reçues, dont un raisonnement ou un événement ultérieurs pourront démonter la signification. Soit la démarche inverse de celle des compilateurs qui auront eu tendance à garder seulement la phase thétique sans en retenir la suite.

Le mortel qui pense un jour faire le mal à l'insu des dieux, il croit en la vilenie et il sera surpris en train d'y croire lorsque la Justice en aura le loisir. (Fr. $835^{7}$ )

Mais si, en homme religieux, j'accomplissais la même chose que les plus irréligieux, comment cela serait-il bien ? ou est-ce que Zeus l'excellent a des intentions contraires à la justice? (Fr. 832 $)$

L'extraction hors du contexte confère aux fragments une tonalité particulière, qui va dans le sens d'une éthique de la piété récupérable par les catholiques. Dans les deux cas se forme pourtant une réflexion sur la justice, qui tend à devenir une valeur transcendante au divin même (voir 832) : la Justice se surimpose à Zeus, elle n'en est pas un attribut, on est donc loin de la monothéologie médiévale, malgré certaines apparences trompeuses.

Vis d'espoir et nourris-toi d'espoir. (Fr. 826)

Que tu ouvres les réserves, il ne le réclamait pas. (Fr. 827)

Mieux vaut un bien humble et bénin pour les mortels qu'une maison grossie par une richesse sans religion. (Fr. 825)

Ces trois phrases ont l'avantage de tout dire puisqu'elles ne disent rien. La première peut servir à toute heure. La seconde renvoie aux sirous (que je transpose par " réserves "), lieux de conservation de blé et de vivres. On imaginerait volontiers le propos échappé d'un plaidoyer pro domo, où l'accusé(e) rappelle sa modération passée. Le dernier adage exprime une satisfaction éthique, mais pourrait facilement être renversé dans les combats verbaux auxquels se livrent les personnages d'Euripide.

Si c'était le premier jour de malheur, et si je ne naviguais depuis longtemps sur l'amère peine, bien sûr, je me débattrais, comme le jeune cheval pour la première fois attelé, à l'instant qu'il reçoit le mors - mais je suis usé et rendu docile par les malheurs. (Fr. 821) 


\section{intéressantes, typiquement euripidiennes, où travaillent de concert la réflexion}

Avec le dernier fragnent de cette série, hous touchons de nouveau à des pensées plus philosophique, la langue et le sentiment.

Avec une étonnante rapidité stylistique, Euripide enchaîne deux ensembles métaphoriques en cinq vers. Les deux images de la navigation et du poulain attelé sont courantes, mais elles sont juxtaposées avec une grande maitrise. Pour la première, il faut, je crois, poursuivre le sème au-delà de énaustoloun (« je naviguais ») et entendre par-delà ponoi (« les peines ») pontos (« la mer »), d'où l'explicitation que je tente. La citation de ce vers que fait Cicéron (en latin) me conforte d'ailleurs dans cette hypothèse ${ }^{9}$. La piste du jeune cheval suit immédiatement, dans la comparaison et la valeur métaphorique de katèrtukôs ( « rendu docile »). C'est un vieux marin du malheur, une monture apprivoisée par l'infortune qui parle. Et non pas la simple résignation (« tu verras, avec le temps, on s'y fait »), mais une voix usée jusqu'à la trame, un reste de force employé à dire qu'il est trop tard.

\section{Du paradoxe}

17 En somme, ce qui va au jeune peut n'être plus valable pour le vieux, dans la fluctuation des situations. La vérité n'est pas toute droite, ni objective, ni éternelle, d'où un goût du paradoxe chez Euripide dont témoignent les derniers extraits.

Oui, ceci est inné en la nature de l'homme ; même en cas de mort juste, chacun n'en a pas moins envie de pleurer ses amis. (Fr. 834)

Une ambiguité comparable se trouve dans le présent fragment. Faut-il regretter qu'on pleure ses amis qui sont morts en justes parce que c'est une façon de ne pas honorer leur grandeur ? ou célébrer la puissance pathétique qui passe outre les considérations éthiques? Je penche pour la dernière solution et ce qu'elle nous apprend sur la violence du sentiment.

Qui dit être viril n'est pas digne d'être appelé viril s'il est lâche, et il est malade d'une maladie honteuse. (Fr. 829)

Chez beaucoup d'esclaves, la honte tient dans le nom, mais le cœur est plus libre que chez les non-esclaves. (Fr. 831 ${ }^{10}$ )

... Ou deux évidences qu'on oublie. Les séparations entre masculin et féminin, libre et servile agissent dans la société grecque. Mais Euripide ne manque jamais de considérer ces caractères comme non-définitifs. Être un homme n'est pas un fait empirique, naturel, médical. Un homme a à être un homme, et rien n'est jamais acquis en la matière. Si être un homme signifie avoir du courage, être droit, poursuivre, alors beaucoup d'hommes ne sont pas des hommes (et des femmes, pas davantage) comme le dit ailleurs Oreste à propos d'un Phrygien; et les femmes héroïques sont aussi des hommes, comme le dit le même Oreste de sa sœur Électre ${ }^{11}$. Une logique identique s'applique aux esclaves, et il ne faut pas minimiser la portée de la réflexion. Dans le contexte social de l'Athènes classique, l'affection de la liberté aux douloi est un élément de critique partielle des institutions collectives, formulée du point de vue de la singularitét ${ }^{12}$.

Mais qui sait si vivre n'est pas ce qu'on appelle être mort, et être en vie ce qu'on appelle mourir - à ceci près pourtant : chez les mortels, ceux qui voient le jour souffrent, tandis que les défunts ne souffrent nullement et que leurs malheurs ne s'accroissent pas. (Fr. 833")

Nous terminons par la fin, donc. Le premier segment est un concetto qui liquide le concept et le transforme en notion. La phrase va à l'encontre de la vie et de la mort dans leurs définitions essentielles pour travailler sur un autre sens du sens. Peut-être que la vie n'est 
pas la vie. Euripide n'a pas volé son surnom de skènikos philosophos ${ }^{14}$, mais il vaudrait mieux dire qu'à partir de la philosophie, de la rhétorique, de la sophistique, il propose une nouvelle vérité dans cette Athènes classique ${ }^{15}$. On n'y obéit pas au principe de noncontradiction, on s'autorise des pas de côté. Et si vivre c'était la mort, puisque le bonheur qui, dit-on, est une valeur de la vie, ne se trouve guère que dans le soulagement de la mort? Mais comme on dit aussi que vivre c'est souffrir, il faut alors déduire qu'un mort est bien mort - ce que nous dit la réserve finale. Le détail modifie l'ensemble. On en reste au « qui sait ?» du théâtre qui, répondant au philosophe, ne sait même pas qu'il ne sait pas, mais vit, souffre, meurt, vit.

\section{BIBLIOGRAPHIE}

AELION Rachel, Euripide héritier d'Eschyle, Paris, Les Belles Lettres, 1983, vol. I.

DeLeuZe Gilles, GuATTARI Félix, Capitalisme et Schizophrénie, Paris, Minuit, 2 vol., 1972-1980.

DETIENNE Marcel, Comparer l'incomparable, Paris, Le Seuil, 2000.

-, Les Maitres de vérité dans la Grèce archaïque, Paris, Maspéro, 1967.

EURIPIDE, Opera omnia, édition du texte grec et traduction en latin [collectif], Glasgow, Duncan, 1821 , vol. VII.

-, dans Les Tragiques grecs. Théâtre complet, avec un choix de fragments, traduction Victor-Henri Debidour, Paris, De Fallois/Le Livre de poche, 2000.

GARLAN Yvon, Les Esclaves en Grèce ancienne, Paris, Maspero, 1982.

GOULET Richard (dir.), Dictionnaires des philosophes antiques, vol. II, Paris, CNRS, 2000.

SтовÉE Jean, Sententiae ex thesauris Graecoris delectae..., édition et traduction latine de Conrad Gesner, Froschauer, 1543.

Tragicorum Graecorum fragmenta, édition d'August Nauck revue par Bruno Snell, Hildesheim, Olms, 1964.

Tragicorum Graecorum fragmenta selecta, édition de James Diggle, Oxford, Clarendon Press, 1998.

WEBSTER T.B.L., The Tragedies of Euripides, Londres, Methuen \& $C^{\circ}, 1967$.

\section{NOTES}

1. J'écarte les paraphrases recensées par Nauck. Je délaisse 836 (" des touffes de feu ») et 837 ( des cordes »), qui ne sont que des occurrences de répertoires. Je ne traite pas non plus 830 pour des raisons analogues. En attendant une réforme des éditions complètes des fragments d'Euripide, j'en reste volontairement au texte de Nauck (et je néglige ce que Diggle propose pour $822 \mathrm{~b}$ ). L'ensemble des fragments traduits ici a au moins l'avantage 
d'une unité curieuse : de citations en citations, voilà ce que la tradition voulut qu'on retînt d'une tragédie.

2. Les curieux peuvent aller voir ce qu'en disent Rachel Aélion, Euripide héritier d'Eschyle, vol. I, p. 178-181 et T.B.L. Webster, The Tragedies of Euripides, p. 131-136.

3. Pour réduire l'étrangeté de la phrase « il y avait les trois garçons d'Agénor », on peut remplacer Thasos par Cadmos (leçon de Diggle) ; mais cela ne me paraît pas nécessaire.

4. Même lointainement, ces lignes s'inspirent des réflexions de Marcel Detienne dans Comparer l'in-comparable, et du concept de dé-/re-territorialisation de Gilles Deleuze et Félix Guattari (qu'on peut par exemple trouver dans Capitalisme et Schizophrénie).

5. Sur le sens des tout derniers mots, je suspends mon jugement. Aucune des propositions que recense Nauck ne me convainc vraiment. Une traduction latine de Stobée (1543), elle aussi embarrassée, prend un parti tranché (et sans doute un peu acrobatique) qui ferait ajouter, à la place des points de suspension « et même les tromperies des amis ». Une solution comparable est adoptée dans une édition d'Euripide (1821), qui, avec le même texte que le nôtre, transcrit en latin "Suave autem est amicorum fallaciae."

6. Pour la fin, une éventualité grammaticale : « et j'éviterai ce blâme »- possibilité moins satisfaisante à notre avis, mais qui ne change pas la portée du dicton sur les marâtres.

7. L'expression éph' hèméran peut signifier « un jour », la solution pour laquelle j'opte, ou « jour après jour », voire « chaque jour », ce que comprend la traduction de 1821 (« quotidie »).

8. Fragment délicat à traduire.

9. Cicéron, « Tusculanes » 3, 28, 67 : « nec tam aerumnoso navigassem salo », cité dans l'apparat critique de Nauck.

10. Fragment également traduit par Debidour.

11. Voir Oreste, respectivement vers 1530 et vers 1204 sq.

12. Sur la question des esclaves et la contestation, menée surtout par les sophistes, on se reportera à Yvon Garlan, L'Esclavage en Grèce ancienne (p. 139 sq. pour la critique de l'institution).

13. Fragment également traduit par Debidour.

14. Le point sur cette expression est fait dans l'article de Jean-Louis Flamant sur Euripide à l'intérieur du Dictionnaire des philosophes antiques (notice 139).

15. Ce serait donc une vérité héritant de l'assertion de la poésie ancienne et concurrente de la vérité rationnelle et objective de l'époque classique que décrit Detienne dans ses Maîtres de vérité.

\section{AUTEUR}

\section{LAURENT DUBREUIL}

Laurent Dubreuil termine actuellement une thèse portant sur les « lectures de la possession » à l'Université Bordeaux-III, où il est enseignant. Au Centre d'études féminines de Paris-VIII, il prépare une étude sur l'amitié et le pathos dans la philosophie. 